\title{
Assistência hospitalar na tentativa de suicídio ${ }^{1}$
}

\author{
Beatriz Aparecida Ozello Gutierrez \\ Escola de Artes, Ciências e Humanidades da Universidade de São Paulo, São Paulo, SP, Brasil
}

Resumo: Este artigo discute pontos relevantes que podem colaborar no sucesso da assistência hospitalar direcionada à pessoa com intenção suicida. $O$ risco de suicídio aumenta de acordo com o número de tentativas e também está associado a intervalos de tempo menores entre essas tentativas. Assim, o acolhimento à pessoa com tentativa de suicídio durante a assistência hospitalar é fundamental, pois se realizado com segurança, prontidão e qualidade é possível determinar a aceitação e a adesão do paciente ao tratamento. Essas ações devem estar direcionadas ao cuidado integral prestado à tríade - paciente/família/equipe de profissionais de saúde e da área social. Existem fatores cruciais no alcance da qualidade assistencial que estão atrelados às condições para favorecer a adesão ao tratamento e a criação de uma equipe multidisciplinar com competência de utilizar uma combinação de fatores biológicos, psicológicos e intervenções sociais por meio de educação continuada.

Palavras-chave: suicídio, tentativa de suicídio, assistência hospitalar, multidisciplinaridade.

\section{Introdução}

Nos últimos 45 anos as taxas de suicídio aumentaram $60 \%$ em todo o mundo. Segundo as estimativas do World Health Organization (WHO, 1998) a cada ano cerca de um milhão de pessoas morrem por suicídio. Este está entre as três principais causas de morte na faixa etária de 15-44 anos, em alguns países, bem como é considerado a segunda principal causa de morte no grupo de 10-24 anos de idade. No entanto, esses números ignoram as tentativas de suicídio que são até 20 vezes mais frequentes quando comparadas ao suicídio consumado (WHO, 1998).

O risco de suicídio aumenta de acordo com o número de tentativas e também está associado a intervalos de tempo menores entre essas tentativas. Dentre os pacientes atendidos em setores de emergência por tentativa de autoextermínio, estima-se que $30 \%$ a $60 \%$ tiveram tentativas prévias e que $10 \%$ a $25 \%$ tentarão novamente no prazo de um ano. As taxas de prevalência de tentativas de suicídio ao longo da vida variam de $0,4 \%$ a 4,2\% (Bertolote et al., 2005).

As ocorrências de tentativas de suicídio ou o suicídio consumado atingem milhões de pessoas, considerandose o fato de que a sociedade diariamente sofre prejuízos no âmbito físico, econômico e emocional advindos dessas situações que acontecem em rodovias, ferrovias, metrovias, viadutos, edifícios, instituições públicas e privadas, e domicílios.

1 Texto referente à palestra intitulada "Atendimento hospitalar nas tentativas de suicídio" ministrada na II Jornada do Laboratório de Estudos sobre a Morte do Instituto de Psicologia da Universidade de São Paulo.

* Autor correspondente: biagutierrez@usp.br
Assim, estimular a conscientização das tendências epidemiológicas relacionadas às tentativas de suicídio e lesão autodestrutiva é um dos primeiros e mais importantes passos para o desenvolvimento de estratégias eficazes para a prevenção de comportamentos suicidas recorrentes ou fatais (Ting et al., 2012).

A realização do acolhimento à pessoa com transtorno mental em situação de emergência também é fundamental, pois se realizado com segurança, prontidão e qualidade é possível determinar a aceitação e a adesão do paciente ao tratamento. $\mathrm{O}$ acolhimento representa a mais importante tecnologia de um serviço de emergência, pois possibilita a escuta ativa pelo profissional, favorecendo a empatia, oferecendo-lhe cuidado integral com respostas adequadas e resolutividade, no âmbito intra e extra-hospitalar, isto é, articulando as possibilidades oferecidas na rede de serviços do sistema de saúde e social (Azevedo \& Barbosa, 2007; França, 2005).

Nessa abordagem, durante o acolhimento existem ações que devem ser priorizadas para o alcance desses objetivos visando diminuir tanto o número relacionado às tentativas de suicídio quanto o próprio suicídio. Essas ações devem estar direcionadas ao cuidado integral prestado à tríade - paciente/família/equipe de profissionais da área da saúde e da área social. Assim, este artigo procurará desenvolver aspectos relacionados a essa tríade durante a assistência hospitalar, dividindo-se em cinco partes. A primeira parte trata sobre o cuidado. A segunda aborda a necessidade da integralidade do cuidado prestado no hospital. A terceira contempla a atuação da equipe multiprofissional frente ao paciente e aos familiares. A quarta descreve a avaliação e possibilidades de melhoria das competências da equipe de saúde. E por fim, a última parte se destina à conclusão sobre as reflexões geradas pelo estudo. 


\section{O cuidado}

Para Boff (1999) a essência humana está no cuidado, sendo este o suporte da criatividade, da liberdade e da inteligência, permeada pelos princípios, valores e as atitudes presentes no agir e no viver.

O cuidado na ótica de Silva (1997) floresce da esfera ciência-arte-espiritualidade, e faz parte de um processo de interação dinâmico e contínuo que requer novas habilidades/capacidades dos seres cuidadores, que invadem as capacidades intelectuais/racionais, para incluir o amor, a sabedoria, a compaixão, a solidariedade, a intuição, a criatividade, a sensibilidade, a imaginação e as formas multissensoriais de percepção.

Dentre as formas de cuidar, destacamos a utilização de técnicas da comunicação e de relacionamento terapêuticos para abordagem mais efetiva, incluindo situações de emergência, nas quais há manifestações de intenso sofrimento. Deste modo, é possível evitar técnicas restritivas e proporcionar assistência de maior qualidade, que transcenda o cuidado voltado somente para o corpo, incorporando o cuidado que considere a dimensão existencial, relacional, histórica, cultural e situacional dos pacientes, como sujeitos humanos desejantes (Kondo, Vilella, Borba, Moraes, \& Maftum, 2011).

O caminho para o humanismo está na compreensão dos indivíduos, sendo que a prática da moral e da ética de um homem de bem e da preservação do humano precisa ser buscada dentro do próprio ser humano, pois o conhecimento da ciência é insuficiente para atingir esses requisitos (Money-Kirlei, 1969).

Nesse sentido, Boff (1999) afirma que o cuidado só existe a partir do momento que o cuidador valoriza o indivíduo que necessita de cuidado. Desde então, passa a compartilhar de seu destino, de suas buscas, de seus sofrimentos e de seus sucessos, isto é, de sua vida. Por isso, torna-se coerente nessa relação, o florescer do cuidado humanizado.

Knobel (1999) salienta que a humanização significa cuidar do paciente em sua totalidade, valorizando o contexto familiar e social, respeitando os valores, as esperanças, os aspectos culturais e as preocupações de cada um. Salienta ainda sobre a necessidade de manter e preservar a dignidade do paciente respeitando-o como ser humano, a partir dos princípios morais e éticos.

A partir do momento que se reconhece o outro que está à sua frente e se coloca em seu lugar, imagina-se receber a verdadeira assistência com qualidade. Nesse momento, o profissional de saúde que se vê como um ser humano, que gosta de si mesmo e se valoriza, deixará a mecanização de lado e conseguirá dar o melhor de si para o outro. Assim, a pessoa cuidada integralmente terá a oportunidade de buscar um novo sentido para a sua vida e, tentar encontrar forças para enfrentar essa fase, por mais dolorosa que seja.

\section{A necessidade da integralidade do cuida- do prestado no hospital}

O hospital é conceituado como: "parte integrante de uma organização médica e social, cuja função básica consiste em proporcionar à população assistência médica integral, curativa e preventiva, sob os regimes de atendimento, inclusive o domiciliar, constituindo-se também em centro de educação, capacitação de recursos humanos e de pesquisas em saúde, bem como de encaminhamento de pacientes, cabendo-lhe supervisionar e orientar os estabelecimentos de saúde a ele vinculados" (Brasil, 1977).

Enfatiza-se que é possível controlar alguns problemas existentes na tentativa de suicídio por meio de assistência adequada oferecida no âmbito hospitalar e, assim, garantir qualidade de vida para essas pessoas. Dessa forma, entende-se, no âmbito biopsicossocial, a necessidade da integralidade do cuidado prestado.

Para alcançar a integralidade no cuidado de pessoas, grupos e coletividade é necessário visualizar o paciente como indivíduo histórico, social e político, articulado ao seu contexto familiar, ao meio ambiente e à sociedade no qual se insere, de modo que 0 atendimento integral extrapole a estrutura organizacional hierarquizada e regionalizada da assistência de saúde, prolongando-se pela qualidade real da atenção individual e coletiva assegurada aos usuários do sistema de saúde e pelo compromisso com o contínuo aprendizado e com a prática multiprofissional (Mattos, 2004).

Nessa abordagem, os serviços de saúde devem funcionar atendendo o indivíduo como um ser humano integral submetido a diferentes situações de vida e trabalho, que o levam a adoecer e a desejar morrer.

Na dimensão biopsicossocioespiritual, a assistência da pessoa deverá ser realizada para além de sua doença, sendo que as ações de saúde devem ser combinadas e voltadas ao mesmo tempo para a prevenção e cura ou mesmo para cuidar daqueles que se encontram fora de possibilidades de cura. Isso exige que essa assistência compreenda a erradicação das causas, a diminuição dos riscos e o tratamento dos danos.

Portanto, esperamos que a atenção integral ao paciente hospitalizado possa representar o esforço da abordagem completa e holística de cada profissional que ao assistir o paciente, respeite-o como uma pessoa portadora de necessidades de saúde que, por determinado período de sua vida, está precisando de cuidados hospitalares. Tal abordagem implica em garantir desde o consumo de todas as tecnologias de saúde disponíveis para melhorar e prolongar a vida, até a criação de um ambiente que resulte em conforto e segurança para a pessoa hospitalizada, visando uma vida digna.

O cuidado, nas organizações de saúde em geral, e, em particular, no hospital é por natureza, necessariamente, multidisciplinar e multiprofissional, isto é, depende da conjugação do saber e do trabalho de vários profissionais. Significa dizer que o cuidado recebido e vivenciado pelo paciente é a somatória de pequenos cuidados que vão se complementando, de maneira mais ou menos consciente e negociada, entre os vários trabalhadores que circulam e produzem a vida do hospital. Assim, uma rede de atos, procedimentos, fluxos, rotinas, saberes, em um processo 
de complementação, vai compondo o cuidado em saúde (Cecílio \& Merhy, 2003).

Logo, a integralidade do cuidado deve ser vista em rede, fazendo que a equipe do serviço de saúde, por meio da articulação de suas práticas, consiga escutar e atender, da melhor forma possível, as necessidades advindas do processo de saúde/doença trazidas pelo paciente.

Tal dinâmica existente no cotidiano dos hospitais é um aspecto central cada vez mais a ser considerado na discussão da integralidade e na sua correlação com o processo de gestão. Uma coisa é pensar o trabalho em equipe como a somatória das ações específicas de cada profissional e como linha de montagem do tratamento da doença, tendo a cura como ideal, outra, é pensar arranjos institucionais, modos de operar a gestão do cotidiano sobre a micropolítica do trabalho que resultem em uma atuação mais solidária e concertada advinda do grande número de profissionais envolvidos no cuidado (Cecílio, 2001).

Desse modo, a integralidade do cuidado no hospital como nos demais serviços de saúde passa, necessariamente, pelo aperfeiçoamento da coordenação do trabalho em equipe.

Tendo o hospital como equipamento de saúde pelo qual circulam os mais variados tipos de pessoas, portadoras das mais diferentes necessidades, em diferentes momentos de suas vidas, pode-se imaginar que há outras formas de trabalhar a integralidade. Por exemplo, o momento de alta do paciente que tentou suicídio deve ser pensado como um momento privilegiado para se produzir a continuidade do tratamento em outros serviços, visando à construção ativa da linha de cuidado necessário àquele indivíduo específico, não apenas como uma forma burocrática, que cumpre um papel de contrarreferência. O período da internação pode, inclusive, ser aproveitado para apoiar o paciente na direção de conquistar maior autonomia e na reconstrução e ressignificação do seu modo de viver.

Para Cecílio (2001) as necessidades em saúde devem ser relacionadas à integralidade tanto em suas dimensões macroestruturais (modos de gestão) quanto microestruturais (organização das ações) em saúde.

Pensando na dimensão microestrutural do cuidado ao paciente que tentou o suicídio, tornam-se fundamentais reflexões sobre a sua vulnerabilidade. Ao abordar esta vulnerabilidade individual é necessário compreender os aspectos biológicos, emocionais, cognitivos, atitudinais e referentes às relações sociais desse paciente (Ayres, Calazans, Saletti Filho, \& França Jr., 2006). Quando se pensa, então, na vulnerabilidade individual, inferimos que um ou vários desses aspectos podem causar algum desequilíbrio e afetar o bem-estar do indivíduo.

Considerando a relevância do bem-estar do paciente, é preciso que a equipe de saúde tenha competência técnica e utilize instrumentos de avaliação que permitam um diagnóstico abrangente das condições físicas, psíquicas, sociais e de recursos de saúde, que colaborem no conhecimento da vulnerabilidade desse paciente, estando este em qualquer área de atenção à saúde e, principalmente, se estiver em unidade hospitalar. Capacitado, o profissional será capaz de verificar a existência de possíveis vulnerabilidades nessa população assistida.

\section{A atuação da equipe multiprofissional frente ao paciente e aos seus familiares}

No âmbito hospitalar, este trabalho é realizado por uma equipe multiprofissional, em que se destacam principalmente os profissionais da equipe médica, de enfermagem, de nutricionistas, de psicólogos, de fisioterapeutas, de farmacêuticos e demais trabalhadores de atividades de apoio.

Nas últimas décadas, a introdução de novas tecnologias no trabalho em algumas áreas tem exigido do profissional a utilização de outro tipo de esforço mental para a realização das atividades laborais. Com isso, muitos estudos foram desenvolvidos na tentativa de explicar e solucionar esse novo problema. Os resultados desses estudos indicam a necessidade de se reconhecer a importância das vivências subjetivas na dinâmica do processo saúde/ doença. Ao mesmo tempo, também foram feitos estudos indagando a dominância de princípios e lógicas econômicas que contrariam propriedades de natureza ética.

Lima Júnior e Esthér (2001) ressaltam que apesar do hospital ter como missão salvar vidas e recuperar a saúde dos indivíduos doentes, propicia o desequilíbrio no estado de saúde de seus trabalhadores, devido ao fato de ser um ambiente insalubre e penoso.

Waldow (1998) aponta que os profissionais têm consciência da necessidade do cuidado humano. No entanto, o cuidado técnico impera em alguns setores dentro do hospital.

Os pacientes após a tentativa de suicídio geralmente estão muito fragilizados, alguns demonstram que se sentem incompetentes por não terem alcançado o ato desejado. Já os seus familiares encontram-se assustados com o fato, preocupados com o estado de saúde do paciente, e em alerta para o que possa ocorrer. Por isso, é fundamental a presença de carinho, de delicadeza e de dedicação durante o convívio com esses pacientes e seus familiares.

Diante dos pacientes que tentaram o suicídio, é relevante que o planejamento das práticas de saúde direcione 0 acesso universal aos serviços de saúde almejando a integralidade do cuidado. Dessa forma, é essencial a capacitação dos profissionais de saúde da atenção básica, das unidades de emergência e dos serviços de saúde mental, os quais deveriam se articular de forma organizada e resolutiva dentro da rede social e de saúde (Vidal, Gontijo, \& Lima, 2013).

No espaço de interface do cuidado, sempre existirá novos aspectos de interesse, de trocas afetivas de envolvimento e interação humana (Steiner, 1986). Nesta linha de pensamento, devemos acreditar que o discurso de toda a equipe interdisciplinar, será importante na assistência a estes pacientes e familiares que necessitam de ajuda.

Sabemos que as cinco maiores áreas de prevenção do suicídio relacionam-se à educação e aos programas de 
conscientização para o público em geral e os profissionais; aos métodos de rastreamento para indivíduos de alto risco; ao tratamento para transtornos psiquiátricos; ao acesso restrito a métodos letais e ao papel da mídia (Mann et al., 2005).

$\mathrm{O}$ estudo evidencia a necessidade de se estabelecer processos de educação permanente à equipe de enfermagem para o atendimento em saúde mental. Os entrevistados reconhecem a necessidade de maior conhecimento nessa área para sua prática profissional e de estrutura mais adequada (Buriola et al., 2011).

A educação permanente é uma estratégia para a consolidação do Sistema Único de Saúde (SUS), e recomenda-se que seja realizado um projeto de educação em serviço na área da saúde mental, utilizando-se como método de ensino/aprendizagem a problematização do processo de trabalho, visando a ruptura dos paradigmas das práticas profissionais de modo que teoria e prática não seja uma dicotomia (Kondo et al., 2011). Nessa abordagem, mesmo depois que um paciente tenha concluído suicídio, as causas do suicídio bem como a eficácia do tratamento oferecido, as atividades assistenciais e preventivas podem ser exploradas para tornarem-se uma fonte de aprendizado. Também, deve-se lembrar que os inquéritos retrospectivos ou autópsias psicológicas envolvem a aquisição de informações fornecidas pela equipe, parentes, familiares e amigos de uma pessoa que cometeu suicídio (Beskow, Runeson, \& Asgard 1990; Pouliot \& De Leo, 2006).

Assim, os objetivos desse procedimento visam aumentar o conhecimento que é útil para futuras atividades de tratamento e prevenção, para melhorar a eficácia de rotinas de cuidados de saúde, e para interrogar a equipe de cuidados de saúde e ajudá-la a gerenciar o estresse, quando um paciente comete suicídio (Grad, 2009).

Muitas vezes, percebemos que o profissional está despreparado para aproximar-se dos familiares de pacientes que tentaram o suicídio, mesmo que esta aproximação seja apenas no sentido de segurar na sua mão ou então, olhar diretamente nos olhos de uma pessoa que está precisando receber um conforto, um aconchego. Apesar de todo um discurso do assistir o paciente/familiares holisticamente objetivando o cuidado humanizado, esse enfrentamento causa ao profissional angústia e sofrimento.

Observa-se que o contato inicial com a família é pontual, restringe-se apenas à coleta de informações referentes à tentativa de suicídio. Espera-se que nesse momento, os profissionais ofereçam cuidado, apoio, zelo e esclarecimentos, porém isso não acontece. A justificativa dessa falta de acolhimento são as precárias condições estruturais das instituições, pela dinâmica de trabalho estressante das unidades de emergência, pela enorme demanda de pacientes nestes setores e ainda pelo despreparo profissional para atuar em situações mais complexas (Buriola et al., 2011).
Salientamos que os familiares, pessoas próximas e entes queridos dos pacientes podem desempenhar um papel importante na prevenção do suicídio, pois são capazes de ajudar os profissionais de saúde na detecção precoce e no gerenciamento do paciente em risco de suicídio (Barrero, 2008, Ram, Darshan, Rao, \& Honagodu, 2012).

A fim de atingir este objetivo, toda a família deve ser informada quanto à prevenção do suicídio, pois é uma causa evitável de morte (Barrero, 2008). Existem quatro medidas essenciais que os familiares precisam saber em relação à prevenção: nunca deixar o paciente sozinho; verificar se todos os familiares estão cientes da tentativa de suicídio e da necessidade de colaborar na vigilância e no fornecimento do apoio emocional ao paciente e contactuar uma instituição de saúde mental para que o paciente receba um atendimento profissional especializado (Barrero, 2008).

Entende-se que o suicídio possui duas vertentes de análise constituídas por fatores relacionados aos contextos sociais e interpessoal nos quais o paciente está inserido, isto é, determinantes intrínsecos e extrínsecos. Por isso, para reduzir o número de suicídios é necessária a presença de ações intersetoriais de enfrentamento contempladas por abordagem multidisciplinar e transdisciplinar (Bezerra Filho et al., 2012). Nesse sentido, a estratégia de estudo de caso torna-se enriquecedora e propicia o alcance da excelência da qualidade da assistência prestada.

No entanto, precisamos nos conscientizar que em alguns casos podem ocorrer o suicídio. Nesta situação, a equipe de saúde deve entrar em contato com a família do paciente, de preferência na primeira semana após o ato suicida, e oferecer apoio emocional para adultos e crianças. As famílias também devem ser informadas sobre a existência de organizações que ajudam os sobreviventes do suicídio (Andriessen, 2009).

Nessa abordagem, é essencial a existência de vínculo entre a tríade - paciente/família/profissionais de saúde - pois essa relação facilitará a integralidade do cuidado.

\section{Avaliação e possibilidades de melhoria das competências da equipe de saúde}

Pacientes suicidas em crise são, muitas vezes, assistidos pelos profissionais de saúde mental, quer após uma tentativa de suicídio ou quando atormentado por ideia suicida grave. Em ambos os casos, um exame diagnóstico rigoroso deve ser realizado. Mesmo que esta primeira avaliação ocorra no departamento de emergência de um hospital geral, é importante que se realize a entrevista em uma área isolada e em uma atmosfera de empatia. Se possível, a informação dada pelo paciente deve ser confirmada com fontes colaterais, incluindo a família (Wasserman et al., 2012). 
Na prática, existem vários tipos de intervenção utilizados por profissionais que trabalham juntos para reduzir o risco de suicídio. A intervenção em crise deve ser intensiva, rápida e precoce. A avaliação do potencial para o suicídio é a primordial, pois ao tomar consciência da crise pode levar a uma diminuição do risco de agir a curto, médio e longo prazo (Ionita, Flórea, \& Courtet, 2009).

Para minimizar a probabilidade de futuras tentativas de suicídio, os hospitais devem colocar em prática a capacitação profissional relacionada ao cuidado ao paciente com risco de suicídio. Por exemplo, os pacientes com diagnóstico de risco de vida que se apresentarem agitados, desanimados, retraídos ou forem portadores de dor intratável serão candidatos para rastreio de suicídio (Ballard et al., 2008).

Em estudo realizado com objetivo de identificar as características e potencialidades das necessidades de três grupos diferentes de pacientes - grupo internado em enfermaria psiquiátrica (grupo HIPW), grupo de centro de cuidados intensivos (grupo HICCC) e grupo não hospitalizado (NH grupo) -, obteve-se que o grupo de pacientes da HICCC precisa de psiquiatras, assistentes sociais e psicoterapeutas clínicos para iniciar imediatamente um cuidado integral por uma equipe multiprofissional. $\mathrm{O}$ grupo HIPW necessita de educação psicológica para evitar a repetição de tentativas de suicídio, de equipe qualificada para realização do tratamento físico e ainda, equipe com habilidade de gestão na ala psiquiátrica. Já os pacientes do grupo NH precisam de um sistema de apoio para convencê-los sobre os riscos de tentativa de suicídio e levar a resolução de problemas com abordagem de questões específicas (Kudo et al., 2010).

Outro estudo com pacientes em risco de suicídio mostrou que a intervenção de uma equipe interdisciplinar foi imprescindível para assegurar uma abordagem terapêutica integral, capaz de cumprir também o papel de mediadora do serviço entre os demais recursos da rede sanitária e social, cabendo à equipe a função de interlocutora com a clientela, cujas necessidades envolviam família, trabalho, justiça e outras situações (França, 2005).

A avaliação adequada do risco de suicídio pode ser realizada por meio de um modelo que deve incluir uma avaliação abrangente das características demográficas, fatores estressores recentes e diagnóstico psiquiátrico. Esse modelo fornece uma estrutura simples que pode ser usado para educar os professores, orientadores, policiais, líderes espirituais e profissionais de saúde, isto é, profissionais de diversas áreas podem aprender a estar atentos a esses domínios e ser ágeis em situações críticas (Overholser, Braden, \& Dieter, 2012).

Além disso, o apoio e a intimidade que, muitas vezes, são fornecidos por meio de um relacionamento marital podem desempenhar um papel importante na proteção de indivíduos em períodos de estresse emocional extremo e ajudar a reduzir o risco de uma crise suicida (Overholser et al., 2012).

A educação continuada da equipe de saúde mental e o exame de rotinas são necessários também, a fim de gradual e constantemente melhorar o tratamento e as estratégias de prevenção de suicídio por meio de tratamento psiquiátrico (Wasserman \& Wasserman, 2009).

Estudos recentes discutem a importância de programas de capacitação médica na atenção primária visando garantir o tratamento adequado aos pacientes com sintomas depressivos e comportamento suicida (Hegerl, Althaus, Schmidtke, \& Niklewski, 2006; Szanto et al., 2007; Roskar et al., 2010). Por isso, educar profissionais de saúde para a prevenção de suicídio é um método eficaz baseado em evidências (Mann et al., 2005). Logo, a educação continuada regular deve ser ministrada a todos os psiquiatras e aos profissionais de saúde que trabalham em atendimento psiquiátrico, com foco nos novos desenvolvimentos do conhecimento e sobre os aspectos éticos e legais (Ramberg \& Wasserman, 2004; Wasserman et al., 2012).

Nessa linha de pensamento, observamos que os processos de educação permanente devem estar atrelados aos processos educativos formais (residências, especializações e capacitações) e ainda, aos processos de aprendizagem vivenciados no cotidiano do trabalho, frente ao contato e troca de experiências multiprofissionais com os pacientes (Bonfim, Bastos, Góis, \& Tófoli, 2013). Assim, iniciam-se mudanças de paradigmas na ideologia do cuidar, pois atualmente, além do conhecimento adquirido nas instituições acadêmicas, institui-se a valorização da experiência advinda tanto do cuidar do paciente quanto de sua família.

No momento em que os profissionais de saúde assistem os seres humanos, eles se deparam diante de sua própria existência em vários aspectos - saúde ou doença, conflitos e frustrações. Desta maneira, na tentativa de evitar o contato com esses fenômenos utilizam-se de subterfúgios, como mecanismos rígidos de defesa, que podem causar-lhes danos tanto na esfera profissional como na pessoal (Martins, 2001).

Um ato suicida não deve ser considerado necessariamente como reflexo de fracasso profissional na identificação, avaliação ou na intervenção terapêutica, pois se os profissionais cumpriram suas responsabilidades relacionadas à avaliação cuidadosa quanto ao risco de suicídio, à atuação profissional no tratamento baseado em evidências e ao planejamento do monitoramento da pessoa com risco suicida, eles poderão manter-se tranquilos e cientes de que os fatores relacionados à competência profissional da equipe foram cumpridos. Pensando assim, a equipe profissional se conscientiza de que o aspecto desencadeador do suicídio encontrava-se fora de sua governança.

\section{Conclusão}

O suicídio e sua tentativa podem ser prevenidos por meio de tratamentos adequados. Porém, nem sempre existe 
a possibilidade dos médicos atuarem na previsão de risco suicida o mais rapidamente possível, pois muitas vítimas de suicídio, especialmente as mais velhas, morrem no seu primeiro ato suicida, logo, a coleta de informações sobre os fatores de risco de suicídio ficam prejudicadas. Também o suicídio, em alguns casos, não pode ser evitado devido à adesão ineficaz ao tratamento. Nessa abordagem, fatores cruciais no alcance de um bom resultado estão atrelados às condições para favorecer a adesão ao tratamento e a criação de uma equipe multidisciplinar com competência de utilizar uma combinação de fatores biológicos, psicológicos e intervenções sociais (Wasserman \& Wasserman, 2009).

Dessa maneira, os profissionais precisam considerar os aspectos biopsicossociais para que estes permaneçam engrenados para conseguir revitalizar a chama da vida dos pacientes com ideia suicida. No entanto, é fundamental que a equipe de saúde mental possua habilidade, conhecimento e atitude, isto é, sejam eficientes ao assistir o paciente de risco de suicídio e seus familiares. Assim, faz-se necessária a capacitação profissional dessa equipe.

Além da assistência destes profissionais, também devemos considerar que os familiares e pessoas próximas ao paciente são elementos-chave que podem colaborar na prevenção do suicídio.

Finalmente, a intervenção de uma política de saúde, instituindo a utilização de um método padronizado à assistência integral prestada à tríade paciente/familiares/equipe pode ter implicações importantes no que diz respeito à prestação de cuidados à saúde, coordenação de cuidados, alocação de recursos humanos e financeiros destinados aos temas: tentativa de suicídio e suicídio.

\section{Hospital care in suicide attempts}

Abstract: It is relevant to discuss points that can help in the success of hospital care directed to the person with suicidal intents. The risk of suicide increases with the number of attempts. Thus, the reception given to this person during hospital care is crucial, because, if done safely, with promptness and quality, it is possible to determine acceptance and adherence to the treatment. These actions must be directed to the comprehensive care provided to the triad (patient/family/team of professionals). There are crucial factors for achieving quality of care which are tied to conditions to optimize adherence to treatment and the creation of a multidisciplinary team competent to use a combination of biological, psychological and social interventions through continuous education.

Keywords: suicide, suicide attempt, hospital care, multidisciplinarity.

\section{Assistance hospitalière de soins de tentative de suicide}

Résumé: Il s'agit de discuter des questions pertinentes qui peuvent contribuer à la réussite des soins hospitaliers adressés à la personne avec l'intention suicidaire. Le risque de suicide augmente avec le nombre de tentatives. Ainsi, I'hôte de cette personne au cours de soins hospitaliers est crucial, car si cela se fait en toute sécurité, la rapidité et la qualité est possible de déterminer l'acceptation et l'adhésion au traitement. Ces actions doivent être adressées à la prise en charge globale prévue à la triade - le patient / famille / équipe de professionnels. Il y a des facteurs cruciaux dans la réalisation de la qualité des soins qui sont liés aux conditions d'optimiser l'adhérence au traitement et à la création d'une équipe multidisciplinaire qui acquiert la compétence d'utiliser une combinaison d'interventions biologiques, psychologiques et sociaux à travers la formation continue.

Mots-clés: suicide, tentative de suicide, soins hospitaliers, multidisciplinarité.

\section{Atención hospitalaria en intento de suicidio}

Resumen: Este artículo trata de discutir temas relevantes que pueden contribuir al éxito de la atención hospitalaria dirigida a la persona con intención de suicidio. El riesgo de suicidio aumenta con el número de intentos y también está asociado a menores periodos de tiempo entre éstos. Por lo tanto, la acogida a esta persona durante la atención hospitalaria es crucial, porque si se hace con seguridad, rapidez y calidad es posible determinar la aceptación y adhesión al tratamiento. Estas acciones deben ser dirigidas a la atención integral a la tríada - paciente/familia/equipo de profesionales de salud y área social. Hay factores decisivos para llegar a la calidad de la atención que están vinculados a las condiciones para optimizar la adhesión al tratamiento y la creación de un equipo multidisciplinario que adquiere la facultad de utilizar una combinación de intervenciones biológicas, psicológicas y sociales a través de la educación continua.

Palabras clave: suicidio, intento de suicidio, atención hospitalaria, multidisciplinaridad. 


\section{Referências}

Andriessen, K. (2009). The role of volunteer organizations in suicide prevention. In D. Wasserman \& C. Wasserman (Eds.), Oxford textbook of suicidology and suicide prevention: A global perspective. Oxford: Oxford University Press.

Ayres, J., Calazans, G. J., Saletti Filho, H. C., \& França Jr., I. (2006). Risco, vulnerabilidade e práticas de prevenção e promoção de saúde. In G. Campos, M. C. S Minayo, M. Akerman \& M. Drumond Jr. (Eds.), Tratado de saúde coletiva (pp. 375-417). São Paulo, SP: Hucitec.

Azevedo, J. M. R., \& Barbosa, M. A. (2007). Triagem em serviços de saúde: percepção dos usuários. Revista de Enfermagem da UERJ, 15(1), 33-9.

Ballard, E. D, Pão, M., Horowitz, L., Lee, L. M., Henderson, D. K., \& Rosenstein, D. L. (2008). Aftermath of suicide in the hospital: Institutional response. Psychosomatics, 49, 461-469.

Barrero, S. A. P. (2008). Preventing suicide: A resource for the family. Annals of General Psychiatry, 7(1). Recuperado de http://www.annals-general-psychiatry. com/content $/ 7 / 1 / 1$

Bertolote, J. M., Fleischmann, A., De Leo, D., Bolhar, J., Botega, N., Silva, D., . . . Wasserman, D. (2005). Suicide attempts, plans, and ideation in culturally diverse sites: The WHO SUPRE-MISS community survey. Psychological Medicine, 35, 1457-65.

Beskow, J., Runeson, B., \& Asgard, U. (1990). Psychological autopsies: Methods and ethics. Suicide Life Threatening Behavior, 20, 307-23.

Bezerra Filho, J. G., Werneck, G. L., Almeida, R. L. F., Oliveira, M. I. V., \& Magalhães, F. B. (2012). Estudo ecológico sobre os possíveis determinantes socioeconômicos, demográficos e fisiográficos do suicídio no Estado do Rio de Janeiro, Brasil, 1998-2002. Cadernos de Saúde Pública, 28(5), 833-44.

Boff, L. (1999). Saber cuidar. Ética do humano - compaixão pela terra (8a ed.). Petrópolis, RJ: Vozes.

Bonfim, I. G., Bastos, E. N. E., Góis, C. W. L., \& Tófoli, L. F. (2013). Interface, 17(45), 287-300.

Brasil. (1977). Conceitos e definições em saúde. Portaria n. 30 - Bsb, de 11 de fevereiro de 1977. Ministério da Saúde. Brasília.

Buriola, A. A., Arnauts, I., Decesaro, M. N., Oliveira, M. L. F., \& Marcon, S. S. (2011). Assistência de enfermagem à família de indivíduos que tentaram o suicídio. Escola Anna Nery Revista de Enfermagem, 15(4), 710-716.

Cecílio, L. C. O. (2001). As necessidades de saúde como conceito estruturante na luta pela integridade e eqüidade na atenção em saúde. In R. Pinheiro \& R. A Mattos (Orgs.), Os sentidos da integralidade na atenção $e$ no cuidado à saúde (pp. 113-126). Rio de Janeiro: ABRASCO.

Cecílio, L. C. O., \& Merhy, E. E. (2003). Integralidade do cuidado como eixo da gestão hospitalar. Campinas, SP. (Trabalho não publicado)
Courtet, R. P. (2009). Prise en charge de la crise suicidaire. L'Encéphale, (Supplément 4), S129-S132.

França, I. G. (2005). Reflexões acerca da implantação e funcionamento de um plantão de emergência em saúde mental. Psicologia: Ciência e Profissão, 5(1), 146-63.

Grad, O. T. (2009). Therapists as survivors of suicide loss. In D. Wasserman \& C. Wasserman (Eds.), Oxford textbook of suicidology and suicide prevention: A global perspective (pp. 609-613). Oxford: Oxford University Press.

Hegerl, U., Althaus, D., Schmidtke, A., \& Niklewski, G. (2006). The alliance against depression: 2-year evaluation of a community-based intervention to reduce suicidality. Psychological Medicine, 36, 1225-33.

Knobel, E. (1999). Condutas no paciente grave (2a ed). São Paulo, SP: Atheneu.

Kondo, E. K., Vilella, J. C., Borba, L. O., Moraes, M. R., \& Maftum, M. A. (2011). Abordagem da equipe de enfermagem ao usuário na emergência em saúde mental em um pronto atendimento. Revista da Escola de Enfermagem da USP, 45(2), 501-507.

Ionita, A., Florea, R., \& Courtet, P. (2009). Prise en charge de la crise suicidaire. L'Encéphale, (Supplément 4), S129-S132

Kudo, K., Otsuka, K., Endo, J., Yoshida, T., Isono, H., Takehito Yambe, T., . . Sakai, A. (2010). Study of the outcome of suicide attempts: Characteristics of hospitalization in a psychiatric ward group, critical care center group, and nonhospitalized group. BMC Psychiatry, 10(4). Recuperado de http://www.biomedcentral.com/1471244X/10/4

Lima Junior, J. H. V., \& Esther, A. B. (2001). Transições, prazer e dor no trabalho de enfermagem. Revista de Administração Empresas, 41(3), 20-30.

Mann, J. J., Apter, A., Bertolote, J., Beautrais, A., Currier, D., Haas, A., . . Hendin, H. (2005). Suicide prevention strategies: A systematic review. JAMA, 294, 2064-74.

Martins, M. C. F. N. (2001). Humanização das relações assistenciais: a formação do profissional de Saúde. São Paulo, SP: Casa do Psicólogo.

Mattos, R. A. (2004). A integralidade na prática (ou sobre a prática da integralidade). Cadernos de Saúde Pública, 20(5), 1411-16.

Money-Kirlei, R. E. (1969). Psicanálise e ética. In M. Klein, P. Heimann \& R. E. Money-Kirlei (Orgs.), Temas de psicanálise aplicada (pp. 140-162). Rio de Janeiro, RJ: Zahar.

Overholser, J. C., Braden, A., \& Dieter, L. (2012). Understanding suicide risk: Identification of high risk groups during high risk times. Journal of Clinical Psychology, 68(3), 349-56.

Pouliot L., \& De Leo, D. (2006). Critical issues in psychological autopsy studies. Suicide and LifeThreatening Behavior, 36, 491-510.

Ram, D., Darshan, M., S., Rao, T. S., \& Honagodu, A. R. (2012). Suicide prevention is possible: A perception 
after suicide attempt. Indian Journal of Psychiatry, 54(2), 172-176.

Ramberg, I. L., \& Wasserman, D. (2004). Benefits of implementing an academic training of trainers program to promote knowledge and clarity in work with psychiatric suicidal patients. Archives of Suicide Research, 8, 331343.

Roskar, S., Podlesek, A., Zorko, M., Tavcar, R., Dernovsek, M. Z., Groleger, U., . . . Murisic, A. (2010). Effects of training program on recognition and management of depression and suicide risk evaluation for Slovenian primary-care physicians: Follow-up study. Croatian Medical Journal, 51, 237-242.

Silva, A. L. (1997). Cuidado transdimensional: um paradigma emergente. Pelotas, RS: Ed. Universitária.

Steiner, C. M. (1986). Obediência: por que aceitamos o controle dos outros. In O outro lado do poder (pp. 41-9). São Paulo, SP: Nobel.

Szanto, K., Kalmar, S., Hendin, H., Rihmer, Z., \& Mann, J. J. (2007). A suicide prevention program in a region with a very high suicide rate. Archives of General Psychiatry, 64, 914-920.

Ting, S. A., Sullivan A. F., Boudreaux, E. D., Miller, I., \& Camargo Jr, A. (2012). Trends in US emergency department visits for attempted suicide andself-inflicted injury, 1993-2008. General Hospital Psychiatry, 34, $557-565$.
Vidal, C. E. L., Gontijo, E. C. D. M., \& Lima, L. A. (2013). Tentativas de suicídio: fatores prognósticos e estimativa do excesso de mortalidade. Cadernos de Saúde Pública, 29(1), 175-187.

Waldow, V. R. (1998). Cuidado humano: o resgate necessário. Porto Alegre, RS: Sagra Luzzata.

Wasserman, D., \& Wasserman, C. (2009). Oxford textbook of suicidology and suicide prevention: A global perspective. Oxford: Oxford University Press.

Wasserman, D., Rihmer, Z., Rujescu, D., Sarchiapone, M., Sokolowski, M., Titelman, D., . . Carli, V. (2012). The European Psychiatric Association (EPA): Guidance on suicide treatment and prevention. European Psychiatry, 27, 129-141.

World Health Organization. (1998). Primary prevention of mental, neurological and psychosocial disorders. Geneva: World Health Organization; 1998. Recuperado em 12 de setembro de 2013, da World Health Organization. Suicide prevention (SUPRE). Recuperado de, http://www.who.int/mental_health/prevention/ suicide/suicideprevent/en/index.html.

Recebido: 21/09/2013

Revisado: $14 / 11 / 2013$

Aceito: $20 / 03 / 2014$ 